\title{
Article \\ Development and Preliminary Evaluation of a Lower Body Exosuit to Support Ankle Dorsiflexion
}

\author{
Tim Schubert ${ }^{1, *}$, Bettina Wollesen ${ }^{2,3}$ (D) and Robert Weidner ${ }^{1,4}$ (D) \\ 1 Laboratory for Manufacturing Technology, Helmut-Schmidt-University, Holstenhofweg 85, \\ 22043 Hamburg, Germany; robert.weidner@hsu-hh.de or robert.weidner@uibk.ac.at \\ 2 Department of Human Movement Science, University of Hamburg, Mollerstraße 10, \\ 20148 Hamburg, Germany; bettina.wollesen@uni-hamburg.de or bettina.wollesen@tu-berlin.de \\ 3 Biological Psychology and Neuroergonomics, Technical University of Berlin, Fasanenstraße 1, \\ 10623 Berlin, Germany \\ 4 Production Technologies, Institute for Mechatronics, University Innsbruck, Technikerstraße 13, \\ 6020 Innsbruck, Austria \\ * Correspondence: tim.schubert@hsu-hh.de
}

Citation: Schubert, T.; Wollesen, B.; Weidner, R. Development and Preliminary Evaluation of a Lower Body Exosuit to Support Ankle Dorsiflexion. Appl. Sci. 2021, 11, 5007. https://doi.org/10.3390/app11115007

Academic Editor: Philip Fink

Received: 3 May 2021

Accepted: 25 May 2021

Published: 28 May 2021

Publisher's Note: MDPI stays neutral with regard to jurisdictional claims in published maps and institutional affiliations.

Copyright: (c) 2021 by the authors. Licensee MDPI, Basel, Switzerland. This article is an open access article distributed under the terms and conditions of the Creative Commons Attribution (CC BY) license (https:// creativecommons.org/licenses/by/ $4.0 /)$.
Abstract: For patients suffering from drop foot due to weakness of ankle dorsiflexion muscles, an ankle foot orthosis provides increased foot clearance during the swing phase of gait, but often restricts other gait functions, such as plantarflexion. Due to steady progress in the development of lighter and smaller actuator technologies, active wearable devices such as exosuits become relevant for rehabilitation, since they can offer an extended functionality including a more comfortable wear than passive plastic orthosis. The aim of the paper is to present a lightweight exosuit supporting dorsiflexion during gait with autonomous recognition of gait phases and conditions. One main requirement during the iterative development of the exosuit is a non-restrictive function, thus no differences between the assisted and non-assisted gait of a healthy subject should occur. We therefore conducted a pilot biomechanics study using statistical parametric mapping to analyze kinematics of the ankle joint and muscle activity of $\mathrm{m}$. tibialis anterior of nine subjects without any gait anomalies walking with and without the exosuit. The results show no significant difference between with and without support. In contrast to passive orthosis, the developed system could be an enhanced solution to assist patients suffering from drop foot, which should be analyzed in the next step for evaluating the development.

Keywords: exosuit development; dorsiflexion weakness; SPM; biomechanical analysis

\section{Introduction}

Injuries or diseases such as stroke or multiple sclerosis can cause neurological impairments which could be followed by muscle weakness [1,2]. For example, a lesion of the common peroneal nerve can lead to an atrophy of muscles for ankle dorsiflexion, such as $\mathrm{m}$. tibialis anterior. Clinically, the gait pattern of these patients is characterized by a drop foot syndrome, thus an insufficient capacity to raise the foot during the swing phase of the gait cycle, which results in a forefoot contact instead of heel strike during walking. The deficient toe clearance is compensated for by increasing knee flexion and hip adduction $[3,4]$. The pathological gait can lead to an increased risk of falling and ankle injuries.

A common method for rehabilitation and to assist everyday walking activities is the use of ankle foot orthosis (AFO) [5,6]. Many studies examined the influence of AFOs on post-stroke hemiparetic gait with drop foot. Studies found improved toe clearance [7], balance [8-10] and gait velocity [11], which was associated with a more self-confident gait [12], lower fear [13] and lower risk of falling.

From a functional point of view, there are two main categories of mobile AFOs (active and passive). Most solutions on the market are passive and rigid assistive devices, which 
are lightweight but constrain the foot in a raised position and inhibit other gait functions, such as limb generation of forward propulsion [14], although propulsive force is important for patients to maintain gait speed as a common therapeutic goal [15-17]. Due to the stiffness of the material, often plastic or carbon [18,19], the user has to preload the material, in order to release the energy afterwards. In the case of a passive ankle orthosis for assisting the foot lifting, the user needs additional muscle force to deform the material and increase strain that is used in the other direction to support the dorsiflexion. Consequently, patients with a lack of neuromuscular activity may be hindered in generating an adequate propulsion impulse. Although passive orthosis should improve ankle mobility, the stiff structure could lead to a converse effect with immobilization of the ankle joint following muscle atrophy. Regarding the development process, different structural analyses and design methods with various material thickness and shapes have been tested [20] to fit wearers' ankles and feet. Nevertheless, Tyson et al. [13] found no difference between bespoke versus off-the-shelf orthosis for gait impairment parameters or satisfaction of stroke survivors. Especially for wearing the orthosis in daily life, wearing comfort is an important requirement, but due to the material of passive AFOs, they often induce pressure points on the body [21]. Consequently, patients might refuse wearing the aids because of uncomfortable usage.

Besides passive orthosis, active exoskeletal devices have the potential to provide immediate assistance during gait. These active devices with incorporated control (e.g., Huo et al. [22], Kirtas et al. [23]) allow changes in their impedance and therefore offer gait-adapted assistance. In contrast to passive orthosis, the active performance could help with re-educating the neuromotor system and facilitate the rehabilitation process [24]. Nevertheless, many of these devices are characterized by a rigid, heavyweight and overhanging design with parallel acting system joints, where a majority of the weight is located on distal joints [25], which might inhibit the natural freedom of movement [26]. Currently, more novel, lightweight and soft wearable designs, so called exosuits, are demonstrated to overcome the disadvantages of passive orthosis and heavy active devices by combining innovative textiles with lightweight actuators. By the use of soft structures and tensile forces, it is feasible to overcome the limitation of reproducing the complex kinematic structure of the ankle as a parallel-acting kinematic mechanism with the risk of misalignment of mechanical axis and human joint [27]. Thus, exosuits often introduce minimal changes to wearers' natural gait because of a biomimetic design comprising of flexible textiles. Awad et al. [28] present a soft, non-restrictive and active exosuit with the possibility to use in everyday life. It combines support of dorsiflexion and plantarflexion and therefore improves the ankle's swing phase, forward propulsion interlimb asymmetry and energy cost of walking. Ding et al. [29] shows an exosuit with multi-joint assistance, which provides synchronized force to the hip and ankle joint during walking. Besides the usage in rehabilitation, other fields of applications may be considered, as this system can reduce metabolic cost and improve walking efficiency when carrying load.

An important feature of active gait support devices for training or daily use is the ability to adapt to different walking conditions, such as level walking or stair ascent and descent. A lot of existing devices are characterized by a complicated design using many sensors, which requires complex control algorithms [30]. Different control strategies can be found in the literature, such as fuzzy logic control [31], PID (position) control [32], adaptive control [33] and inverse dynamics control [34]. Unfortunately, an increase in complexity of control systems through multiple sensors and impedances does not ensure the best solution for orthotic devices, because they are becoming cost-intensive and not suitable for daily use [35]. Thus, the challenge is to simplify as much as possible and to develop an easy and reliable control algorithm to detect required gait events (e.g., heel strike, toe-off, etc.) and gait modes (e.g., level walking, stair ascent/descent, etc.). Moreover, the gait modes must be recognized as fast as possible to prevent the user from falling.

To combine the advantages of a lightweight passive orthosis and a gait-adaptable active device, we developed an active, soft and compact exosuit with an easy but effective 
control algorithm for supporting the ankle joint during plantarflexion at the push-off phase to improve propulsive force. Additionally, it can provide ankle dorsiflexion torque in swing phase. This paper presents the design of the exosuit and a method for validating a nonrestrictive function as one key requirement when developing assistive devices. Therefore, analysis of ankle, knee and hip kinematics and muscle activation with healthy subjects were conducted while walking with the activated exosuit. The aim of the study was to determine differences in joint kinematics and muscle activation between walking with and without the system. Occurring deviations would reflect joint restrictions, which are typically for passive orthosis and should be excluded to prove the advantage of the exosuit. We hypothesized that joint angle trajectories and electromyography signals do not differ between the conditions due to the biomimetic system design in combination with a high dynamic actuator. Methodically, the purpose was to present an approach to examine differences along the whole gait cycle without restricting discrete parameters such as the maximum, minimum or range of motion, since that can withhold important information.

\section{Exosuit Functionality and Design}

\subsection{Functionality}

The purpose of the developed exosuit is to support and enhance the ankle movements of the sagittal plane during gait. The two main functions include (1) the actuation of the plantarflexion and (2) the actuation of the dorsiflexion. During rehabilitation, it is intended to train a gait-dependent activation of the impaired muscles. Current non-articulated solutions often lower the activity of dorsiflexion muscles due to a "bracing effect", which is often provided from passive orthosis devices made of material with a high degree of stiffness [36]. Additionally, a resistance to plantarflexion makes it also difficult to achieve a sufficient forward propulsion during gait [37]. On the one hand, this exosuit intends to provide an active impulse to the ankle during swing phase, so that it is possible to gain toe clearance without an inhibition of the simultaneous muscle activation. On the other hand, the push-off is supported to achieve a powerful plantarflexion without restricting the movement.

The gait cycle (GC) is illustrated in Figure 1. The gait cycle can be divided into the stance phase from 0 to $60 \%$ GC, during which the leading limb (right side in illustration) has contact with the ground. At the following swing phase, from 60 to $100 \%$ GC, the foot is lifted off the ground and swings forward to the next heel strike. The exosuit supports the ankle at two sub-phases during gait. At the end of the stance phase, the heel lifts up and the forefoot pushes off the ground due to a plantarflexion moment of the ankle. The system actuates and supports this degree of freedom by pulling up the heel until the entire foot takes off and leads to the swing phase of gait cycle. During the first half of swing phase (pprox. $25 \%$ of the gait cycle) the foot is lifted up by a dorsiflexion movement of the ankle joint. Whereas the entire swing phase lasts $\sim 400 \mathrm{~ms}$ [38], the foot is actuated and lifted during the first $50 \%$ in $\sim 200 \mathrm{~ms}$ and is held until heel strike.

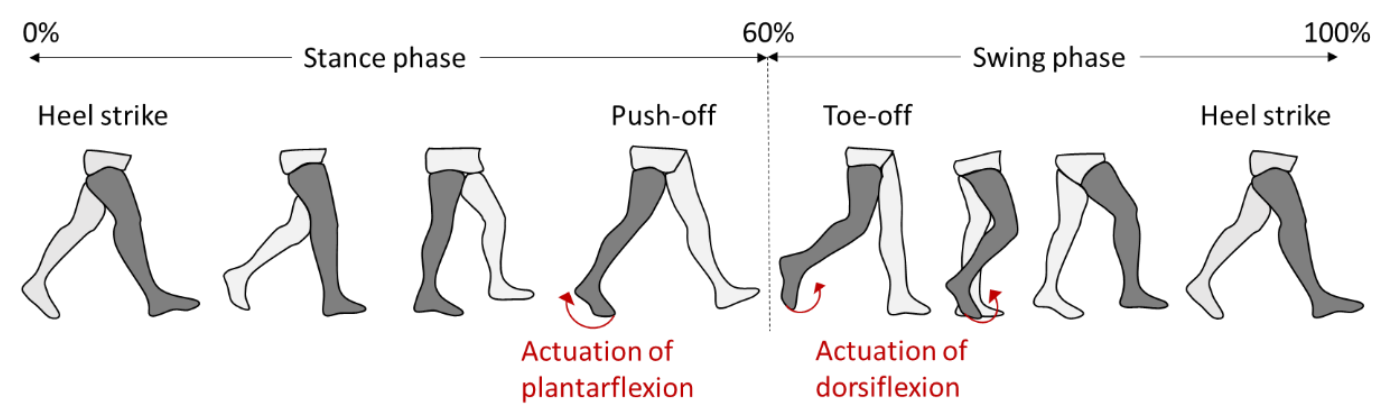

Figure 1. Illustration of supporting function during gait cycle. 


\subsection{Exosuit Design}

The soft and active exosuit does not use any rigid frame structures and may be worn under or over clothing. All components are simply adaptable to different users and are independent from different footwear. Due to the primarily used soft textiles, there are no constraints on the natural passive joint motion. It assists the wearer in plantarflexion at the push-off phase and dorsiflexion during the swing phase of either the left or right foot, which is realized by a pneumatic actuator. The force flows from the actuator, which is located in a hip belt, to the foot by a Bowden tube system. The exosuit contains four main parts: (1) the supporting and force-transmitting structure; and (2) the actuation, (3) the sensory and (4) the control part. The system is illustrated in Figure 2. All main elements are explained in the following sections.

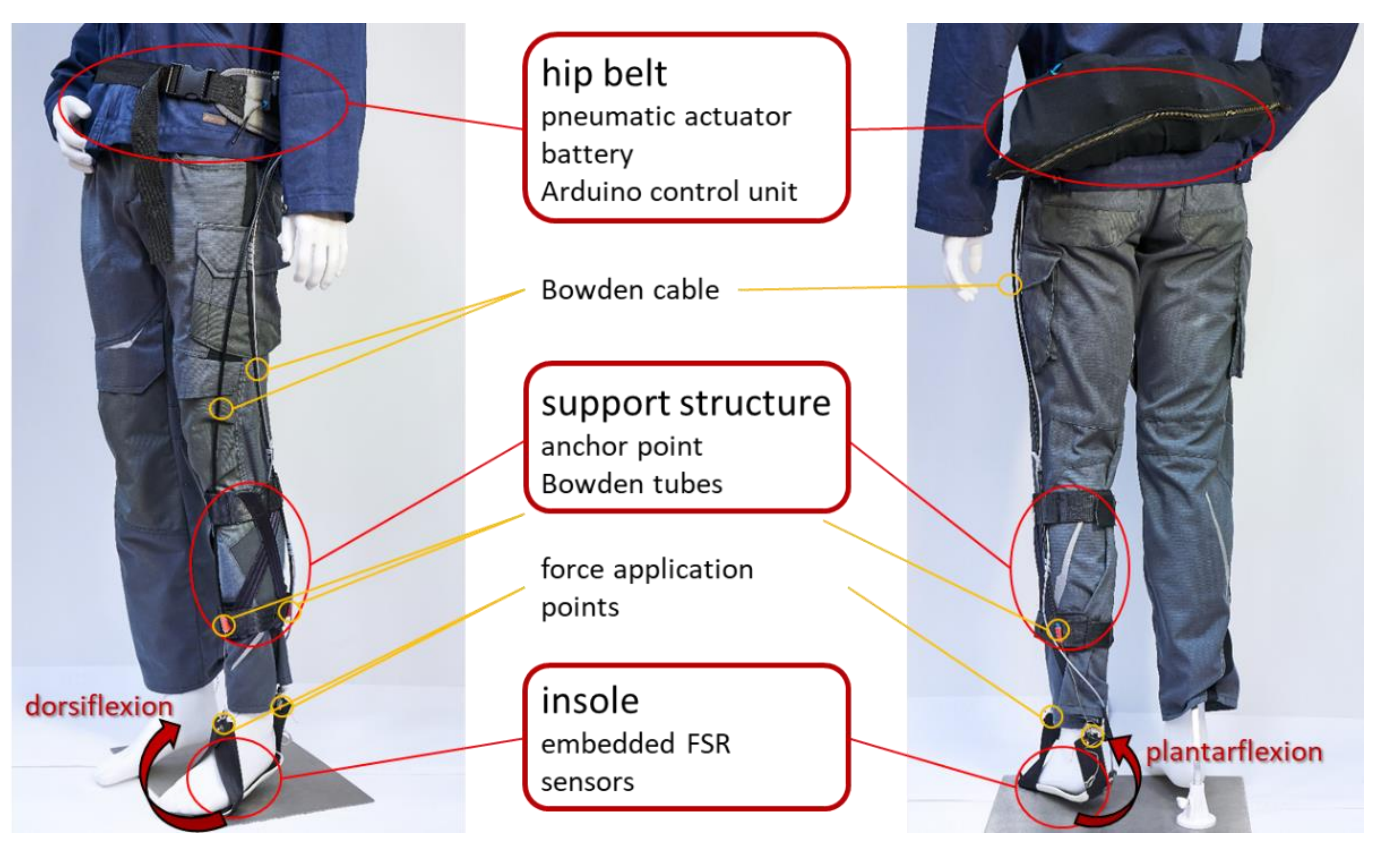

(a) (b)

Figure 2. (a) Frontal view and (b) rear view of underbody exosuit.

\subsubsection{Supporting and Force-Transmitting Structure}

Creating a supporting structure is one of the key challenges in developing an exosuit, as it constitutes a human-suit interface to apply force into the body or body regions in an effective, comfortable and safe manner [39]. The current structure is composed as a biomimetic design and is therefore based on the ligament and tendon structure of the ankle. Whereas tendons are soft tissues which connect muscle and bone to transmit force, ligaments connect bones and stabilize. The tendon of the $\mathrm{m}$. tibialis anterior, as the primary muscle that facilitates dorsiflexion of ankle joint, inserts medial of the first metatarsal bone of the foot. The tendon of the synergist $\mathrm{m}$. extensor digitorum longus inserts at the II-V phalanges. To maintain a stable lever arm, the tendons are held by the extensor retinaculum in place. The antagonists to dorsiflexion are the muscles of triceps surae which transmit force on the Achilles tendon and consequently induce plantarflexion at the ankle. In analogy to the biological structure, the supporting and force-transmitting structure of the exosuit follows a similar mechanism. The supporting structure comprises three main parts:

(1) Artificial tendon-Two Bowden cables are used to transmit the actuation force from the actor-which is located on the hip belt-to the foot and therefore mimic the tendons of the dorsiflexion and the plantarflexor muscles, respectively. The steel cables are stored in a pressure-stable Bowden tube to transmit the force over any curved path. The length of the Bowden cable can be adjusted to fit different leg length; 
(2) Insole-The force-transmission into the foot is realized by a seatbelt webbing loop, which is sewn under an insole in the area of the 1st and 5th caput ossis metatarsalis, where a good transmission of force is given, due to the small soft tissue thickness [40] and a large possible lever arm to the ankle joint. Due to the high stiffness of seatbelt webbing for tensile forces, it is suitable to transmit forces without much elongation. As the webbing loop distributes the force over the entire foot width, it is possible to use only one artificial tendon for dorsiflexion. Regarding different footwear, the use of an insole allows an easy adaptability by providing multiple sizes of insoles, which can be placed in the wearer's shoe and attached to the Bowden cable using a screw lock. To consider users with different gender and anthropometry, we provided three sizes of insoles (small, middle, large);

(3) Webbing-To transmit the force into the lower leg and to maintain a lever arm of about $5 \mathrm{~cm}$ to the ankle joint, a structure of two webbing loops ( $5 \mathrm{~cm}$ width) with diagonal connections was designed. The first loop above the ankle joint acts as tension band wiring and therefore mimics the extensor retinaculum. The Bowden tube is attached to an anchor point on the frontal side of this webbing loop using a 3D-printed adapter which is sewn onto the textile. A second Bowden tube for plantarflexion is attached to the back side. This webbing loop is linked to an upper loop above the calf to transmit the counterforce from the attached Bowden tube to the muscle body of the calf. The requirement is a firmly holding interface to the body. The localization of the human-system interface above the thicker muscle body in combination with anti-slip pads made of cellular rubber on the inner side of the webbing allows a slip-resistant possibility to transmit the force into the body. Moreover, the pads distribute the force to a larger area of the skin and provide a buffer for better wearing comfort.

An illustration of the biomimetic concept and the linkages between the webbing loops of the exosuit and the tendons and ligaments of the ankle are shown in Figure 3.

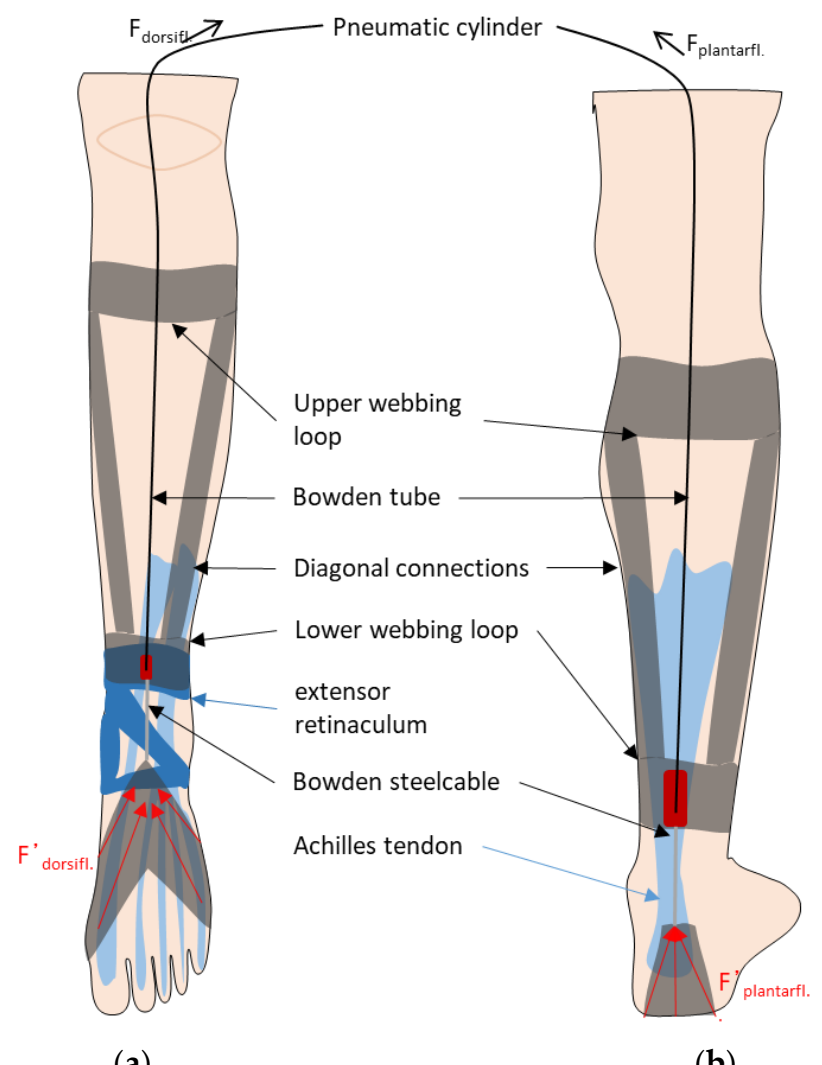

(a)

(b)

Figure 3. Illustration of ankle tendon and ligament mechanism and overlaying exosuit concept of (a) front side of lower leg to induce dorsiflexion and (b) back side of lower leg to induce plantarflexion. 


\subsubsection{Actuation}

The actuation part is composed of a pneumatic double-acting cylinder (stroke: $50 \mathrm{~mm}$, diameter $25 \mathrm{~mm}$, max. $300 \mathrm{~N}$ ), a 5/3 solenoid valve for air injection and release, a Bowden cable which is connected to the cylinder to transmit force and a compressed air tank to operate autonomously. The generated torque actuates the ankle joint in the direction of dorsiflexion and plantarflexion. Due to the double-acting mechanism of the cylinders, it is possible to use one cylinder for actuating two different directions of torque. Furthermore, a pneumatic system ensures high dynamics. This is important due to the short time of about $200 \mathrm{~ms}$ while the foot is lifted in swing phase.

For preventing additional stress to distal joints due to the weight of system components, the actuation module was stored in a hip belt. To locate the majority of the system weight near the centre of the body, it also comprises the $12 \mathrm{~V}$ battery for powering an Arduino control device and the valve for the pneumatic cylinder.

\subsubsection{Sensory}

Detection of ground contact events during gait is ensured by two flexiforce forcesensing resistors (FSR) under the sole, in the area of the 1st caput ossis metatarsalis to determine forefoot contact, as well as under the heel to determine hindfoot contact. The sensors act as on-off foot switches. Due to differing tightness of the worn footwear, the sensors need to be calibrated. Therefore, before every use, the sensors are reset to zero while wearing shoes and lifting the leg off the ground, so that no ground reaction force is being detected.

Additionally, an inertial measurement unit (IMU) is attached laterally at the thigh, to measure the sagittal thigh orientation angle during walking [41]. When standing in an upright position, the thigh angle is equivalent to $0^{\circ}$. With a flexed hip joint of $90^{\circ}$ and the thigh in a horizontal orientation, the thigh angle corresponds to $90^{\circ}$.

\subsubsection{Control}

An Arduino Uno is used for signal processing of the sensory input signals and for control of the actuation output. The force sensors under the foot and the IMU at the thigh are used as input signals for situation detection, whereas the force sensor detects gait events and the IMU differentiates between stair and level walking. The Bang-band control can switch between two states: extension and retraction of the pneumatic actuator. The underlying control scheme is illustrated in Figure 4.

A fixed threshold for the force sensors is selected to detect ground contact. Furthermore, $50 \mathrm{~ms}$ of continuous force is necessary to avoid false contact detection from spontaneous force peaks during walking. This time period was pre-determined by sensitivity analysis with parameter variations. The delay doesn't prevent a timely actuation because of the localization of the FSR forefoot sensor and the underlying time-period starting from the lifted 1st caput ossis metatarsalis (no ground contact on FSR) till the toe-off during the pre-swing phase. The detected ground contact events are associated with either heel strike (loading response), push-off (terminal stance) or toe-off (swing phase). At toeoff, no force is detected neither at heel nor at forefoot, and the thigh angle is controlled to detect current gait mode. As a preliminary experiment has shown, the sagittal thigh angle at toe-off is in a more positive orientation (approx. 30 degree) when walking downstairs compared to level walking (approx. 0 degree). By using the midpoint of 15 degrees as a fixed threshold at toe-off, it is possible to differentiate between level walking and stair descent condition with the feedback of the IMU. Therefore, if the thigh angle is above the threshold at toe-off, the subject is walking downstairs. During stair descent there is not any support of dorsiflexion necessary due to the plantarflexion movement at swing phase, hence there will be no actuation. If the thigh angle is below the threshold, the gait mode switches to level walking. During level walking, there is no actuation at heel strike. At push-off phase, the ankle plantarflexion is supported and during the following swing phase, the dorsiflexion actuation is on. 


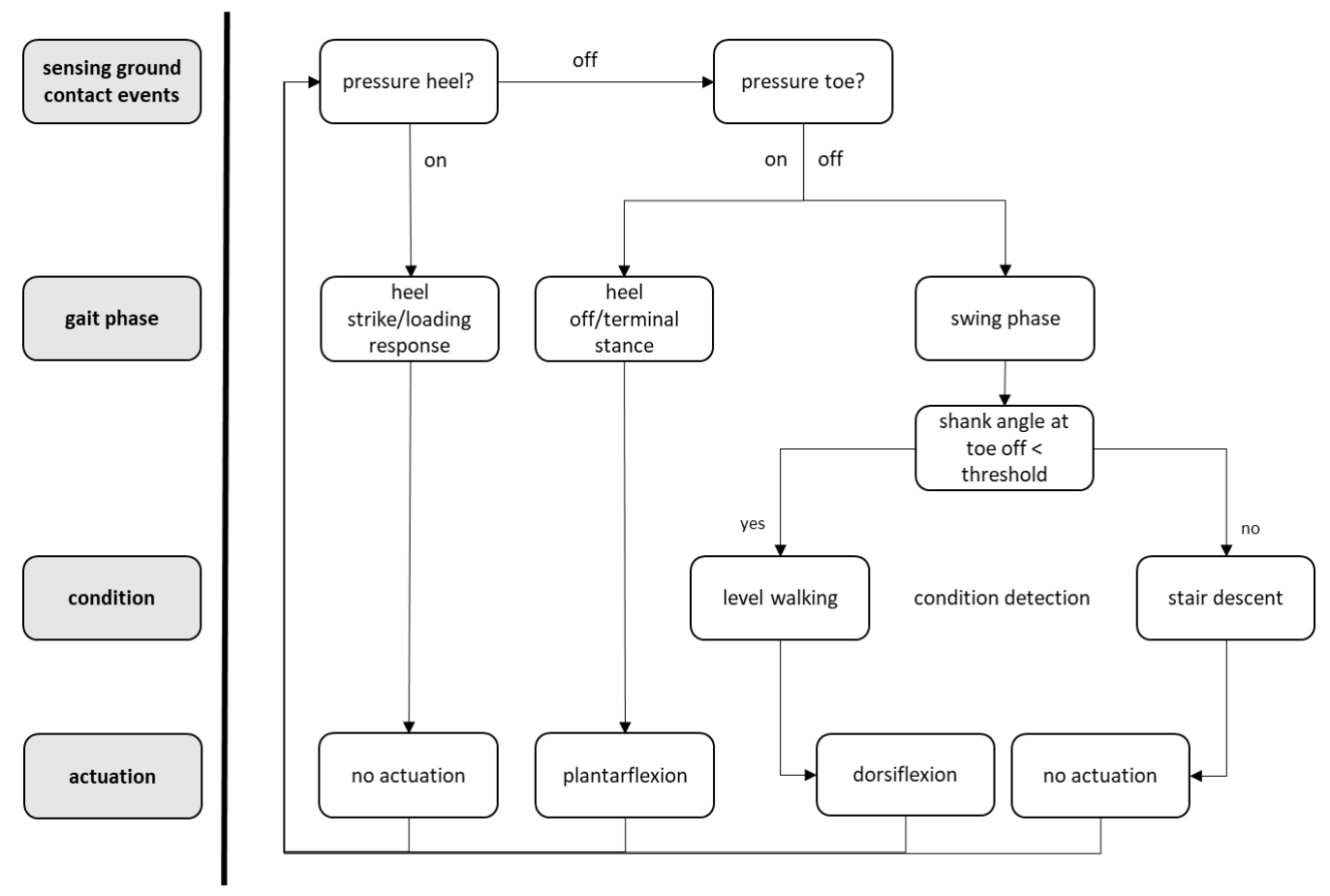

Figure 4. Control scheme.

\section{Preliminary Evaluation}

In order to evaluate the design concept of the exosuit, we conducted a preliminary experiment to ensure a non-restrictive function during gait with the activated system. Using the described lower body exosuit, we measured lower body kinematics and muscle activity of the $\mathrm{m}$. tibialis anterior. In addition, we present the initial feedback and observations from the participants to gain insights into subjective perception, acceptance and usability.

The study was conducted according to the guidelines of the Declaration of Helsinki. Informed consent was obtained from all subjects and they were free to withdraw from the study at any time. A local ethics committee approved the study. The experiments were conducted after explaining the nature and possible consequences of the orthosis.

\subsection{Participants}

Nine neurologically intact subjects ( $n=6$ men; age $31 \pm 6$ years; mass $88 \pm 15 \mathrm{~kg} ; n=3$ women; age $24 \pm 3$ years; mass $59 \pm 1 \mathrm{~kg}$ ) participated in this study. Only participants without any gait abnormalities were considered eligible for the study.

\subsection{Experimental Design}

To measure lower body kinematics during gait, a mobile Xsens MTw Awinda System was used (Xsens Technologies BV, Enschede, Netherlands). This device shows an acceptable to excellent reliability and excellent validity [42]. With a Myon 320 system (Myon AG, Schwarzenberg, Swiss), the muscle activity of the $\mathrm{m}$. tibialis anterior was recorded. The system exhibits good to acceptable reliability [43]. The sensor placement was conducted following SENIAM (Surface Electromyography for Non-Invasive Assessment of Muscles) guidelines. To synchronize both systems, a trigger signal from Xsens was used to mark start and stop into Myon Software.

At the beginning, all subjects underwent a maximum voluntary contraction test for $\mathrm{m}$. tibialis anterior. For Xsens System, a calibration walk of eight steps going back and forth was needed. Afterwards, each participant put the exosuit on the left lower leg and adjusted the size of the hip belt and supporting structure, as well as the length of the Bowden cable to get a sufficient foot lift. Turning the system on, they were able to familiarize with the activated exosuit for five minutes to overcome unwanted protective posture during gait 
and achieve a steady state. They completed a level walkway with 22 strides with and without wearing the exosuit on the left leg while walking with self-selected gait speed and maintaining their usual gait pattern. The with and without support trials were randomized and stopped after the first signs of stumble or any abnormal functions of the exosuit.

\subsection{Data Collection and Analysis}

The data were captured using the provided Xsens Analyze software and the ProEMG Software (2.1.3.6, Myon AG, Schwarzenberg, Switzerland, Win10) for the Myon320 system. The electromyography (EMG) data were normalized to maximum voluntary contractions (MVC), rectified and bandpass-filtered (4th order Butterworth filter; cut-off frequency, 20-200 Hz). While for the EMG device no input for a trigger signal was available, an EMG Sensor was used to capture the trigger output signal of Xsens, which was lowered to $0.5 \mathrm{mV}$ by using a voltage divider. Inside Xsens Analyze, events were marked at heel strikes and toe-offs.

In Matlab (9.5.0.944444, R2018b, MathWorks Inc., Natick, MA, USA, Win10), the kinematic data were cut from start to stop trigger signal, segmented between heel strikes and time-normalized to $0-100 \%$ of gait. For EMG data, the events from Xsens were used to export the muscle activation at swing phase, between toe-off and heel strike. The first and last stride of each trial were removed to analyze the steady state without including accelerating effects at the beginning and end of walking. Therefore, 20 strides for each condition were included to calculate the mean trajectories of the ankle sagittal, frontal and transverse plane, as well as hip and knee sagittal plane angles.

The design of the exosuit regarding the subjective perception, acceptance and usability was determined following the biomechanical measurements. The participants were encouraged to describe their overall impressions on aspects such as the efficiency of ankle assistance when walking with the exosuit, the local perceived discomfort and the size adjustment when putting the system on.

\subsection{Statistical Analysis}

To test the null hypothesis, i.e., no differences for kinematic and EMG data between walking with and without the exosuit, an SPM1d statistical analysis was conducted with the open-source software package from www.spm1D.org (accessed on 3 May 2021) [44] within Matlab. As described by Pataky [44], a between-condition statistical analysis was performed using a paired t-test. The null hypothesis was rejected if the computed SPM $\{\mathrm{t}\}$ value exceeded the critical value. With SPM1d, we analyzed the difference in the mean trajectory of one-dimensional biomechanical data by statistically examining the entire time-series between the conditions. For EMG data, differences during swing phase were investigated. All statistical comparisons were made in SPM1d and based on the Bonferroni correction of alpha for multiple comparisons, $\alpha=0.01$.

\section{Results}

\subsection{Kinematic Data}

Mean kinematic trajectories of the ankle sagittal, transverse and frontal plane, as well as the hip and knee joint in the sagittal plane, are shown on the left side in Figures 5-9. All kinematics of the baseline measurement without the exosuit are illustrated in blue and with exosuit in red, respectively. All angles concern the left leg, on which the exosuit was worn. The gait cycle started with the heel strike and ended with the heel strike of the same leg and next stride. The stance phase, where the foot had contact with the ground, changed into swing phase at about $64 \%$ of gait cycle. The corresponding statistical test is illustrated on the right side of every figure. It indicates the SPM $\{t\}$ curve (black) and the critical value (red, dashed line). By exceeding the critical value, significant differences can be observed throughout the entire gait cycle. In general, the analyzed joint kinematics within the current study did not significantly differ between the walking with and without the exosuit conditions. 
The sagittal plane motion of the ankle describes the dorsiflexion and plantarflexion degree of freedom. Positive values illustrated in Figure 5 correspond to dorsiflexion, where the foot was lifted upwards, and negative values to plantarflexion movement. At heel strike at $0 \%$ of gait cycle and the following stance phase, the angle curves of both conditions follow the same movement without any displacement. From the beginning of the initial swing phase at $64 \%$ of gait cycle, the foot was supported by the exosuit for dorsiflexion movement. At the minimum plantarflexion angle, a mean difference of $-2.23 \pm 7.01$ was noticeable.

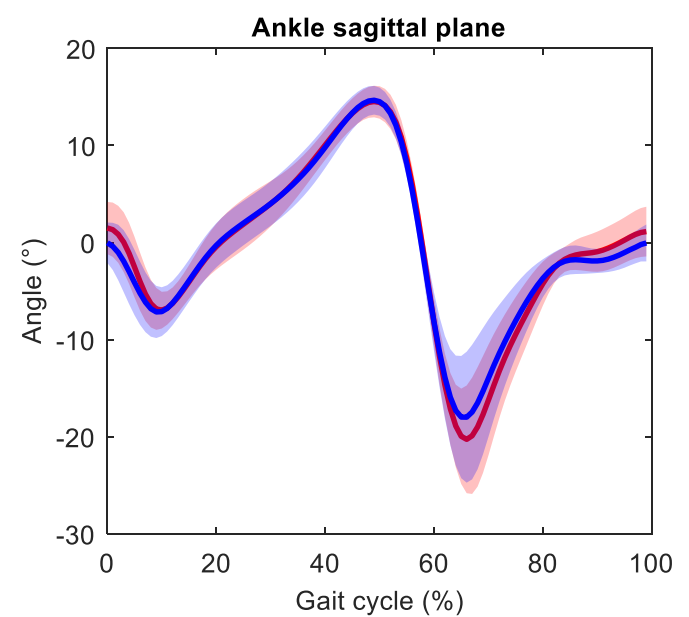

(a)

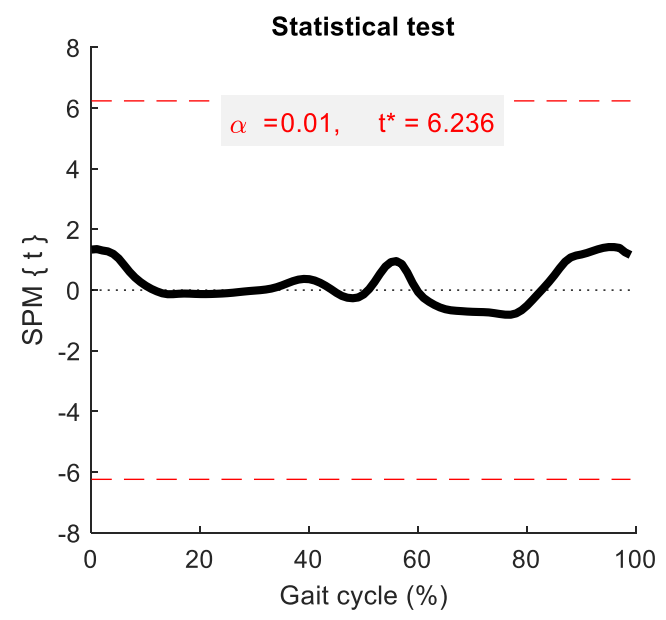

(b)

Figure 5. (a) Mean sagittal ankle angle during gait cycle from heel strike to heel strike with (red) and without (blue) exosuit, and (b) statistical 1D SPM test; * critical t-value.

The ankle transverse plane motion is shown in Figure 6. The degree of freedom for adduction is depicted in the positive direction of the y-axis, and for abduction in the negative direction. The greatest difference between walking with and without the exosuit was at the minimum abduction angle during initial swing phase, of $0.72 \pm 0.89^{\circ}$. Nevertheless, the slight displacements were not significantly different.

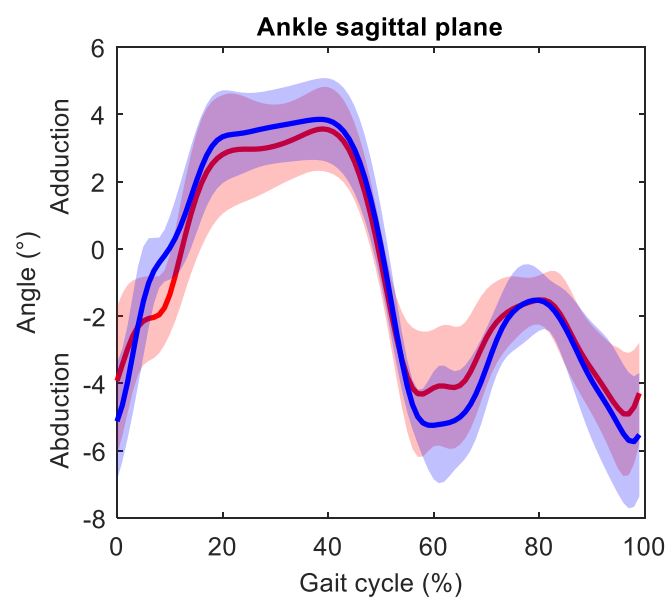

(a)

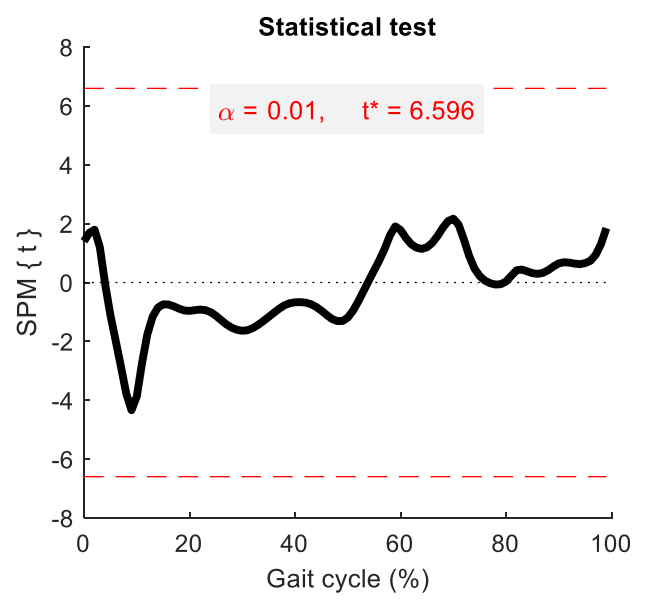

(b)

Figure 6. (a) Mean transverse ankle angle during gait cycle from heel strike to heel strike with (red) and without (blue) exosuit, and (b) statistical 1D SPM test; * critical t-value. 
The mean trajectory of the ankle frontal plane is shown in Figure 7. Positive values indicate the inversion movement, whereas negative values present eversion movement. The maximal difference between the two conditions was $3.43 \pm 1.62^{\circ}$ at $64 \%$ of gait cycle (initial swing phase). Additionally, walking with the exosuit had a slightly larger variance.

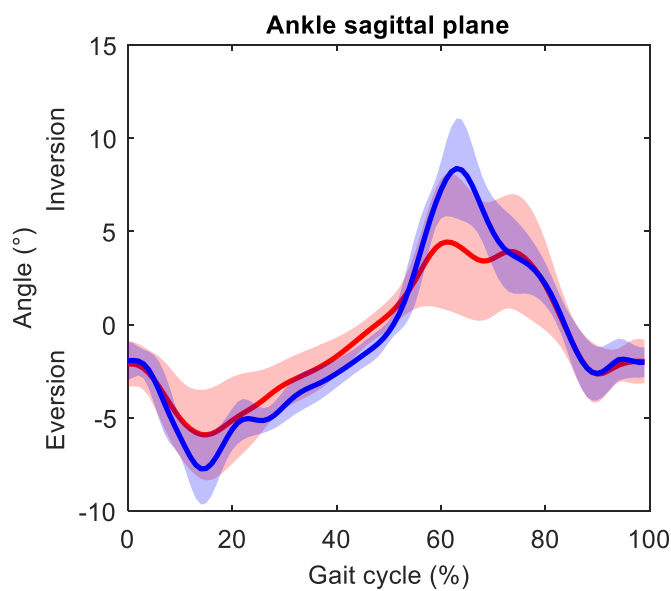

(a)

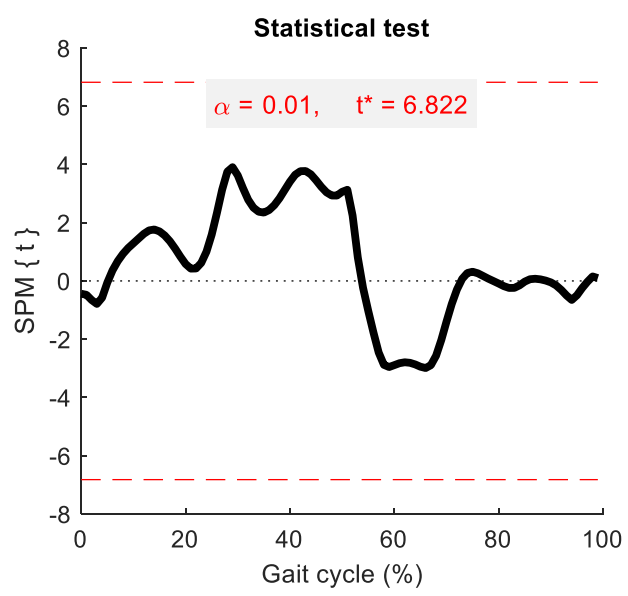

(b)

Figure 7. (a) Mean frontal ankle angle during gait cycle from heel strike to heel strike with (red) and without (blue) exosuit, and (b) statistical 1D SPM test; ${ }^{*}$ critical t-value.

The sagittal angle curves for knee and hip are shown in Figures 8 and 9, which summarize the flexion of the corresponding joint with positive values and an extension movement with negative values. There was no difference between walking with and without the exosuit, neither for the mean trajectory nor for the variance.

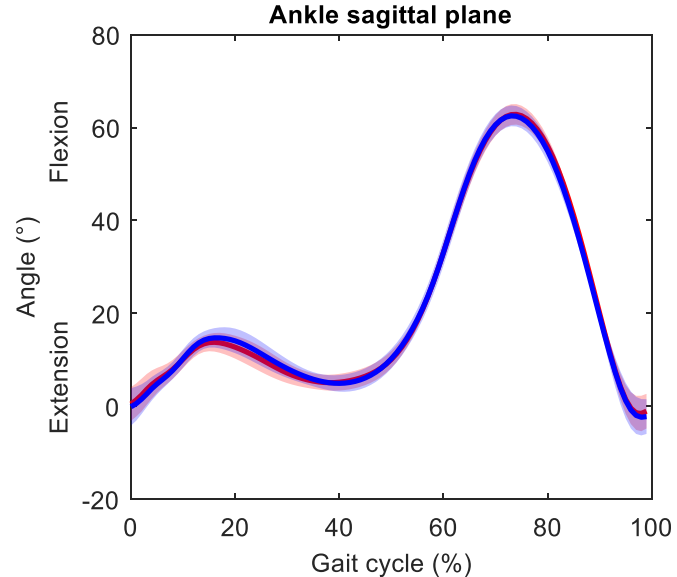

(a)

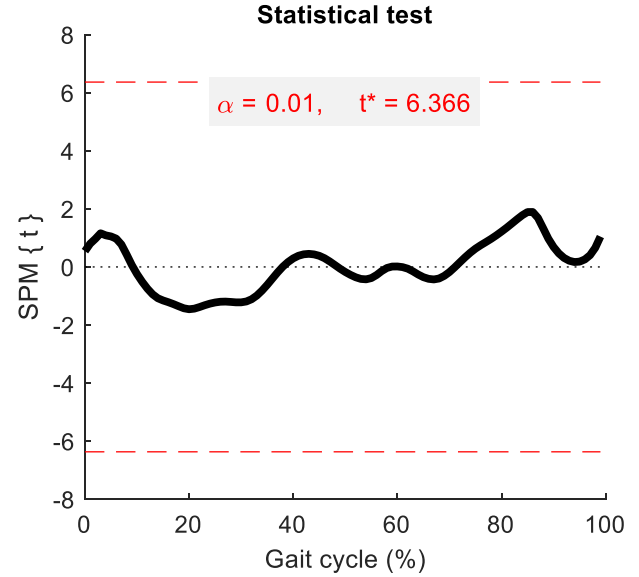

(b)

Figure 8. (a) Mean sagittal knee angle during gait cycle from heel strike to heel strike with (red) and without (blue) exosuit, and (b) statistical 1D SPM test; ${ }^{*}$ critical t-value. 


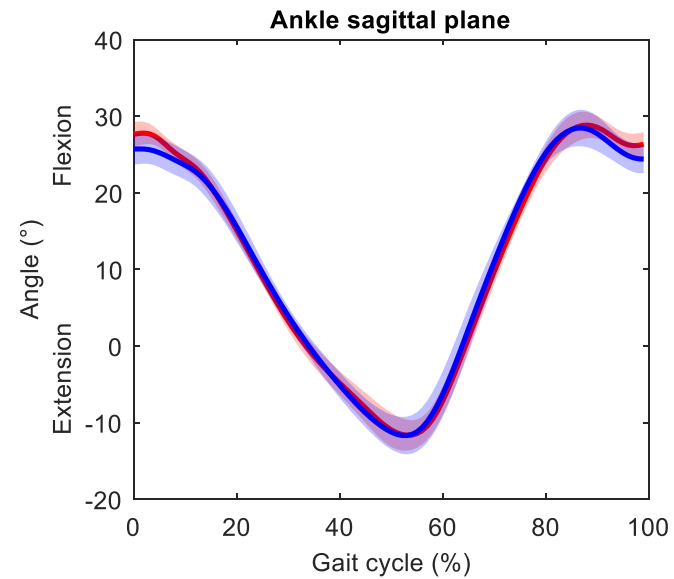

(a)

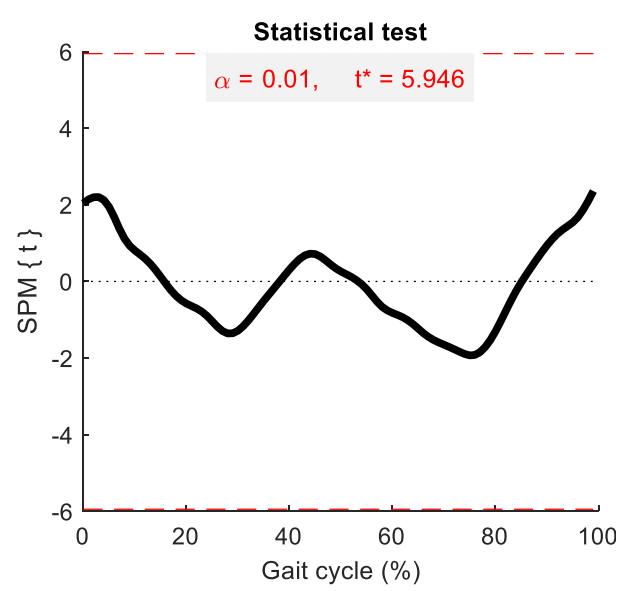

(b)

Figure 9. (a) Mean sagittal hip angle during gait cycle from heel strike to heel strike with (red) and without (blue) exosuit, and (b) statistical 1D SPM test; * critical t-value.

\subsection{EMG Data}

The results for muscle activation of $\mathrm{m}$. tibialis anterior during swing phase are shown in Figure 10. The mean muscle activation during swing phase when walking without the exosuit was $16 \%$ of MVC in comparison to $15 \%$ of MVC when walking with the exosuit. The difference in EMG for the entire swing phase was not significant. The variance for the initial situation without the exosuit was smaller for the exosuit condition.

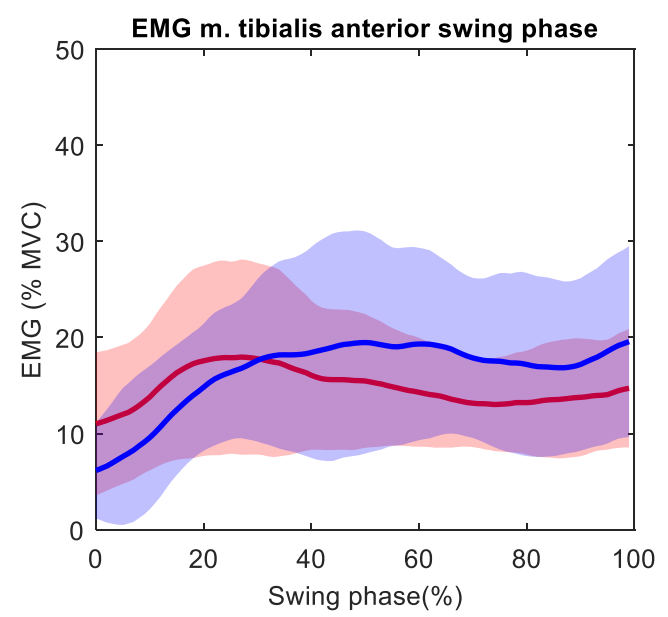

(a)

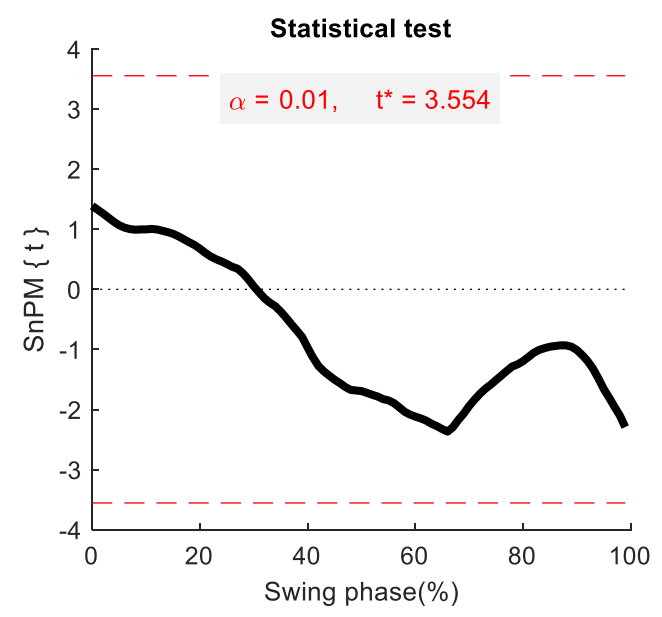

(b)

Figure 10. (a) Mean EMG curve of m. tibialis anterior with (red) and without (blue) wearing exosuit, and (b) statistical 1D SnPM test; ${ }^{*}$ critical t-value.

\subsection{User Feedback}

In general, the participants reported an easily usable exosuit with high wear comfort. They declared that the soft textile elements of the human-system interfaces prevented uncomfortable pressure points on the body. The participants were able to put on and take off the device in less than two minutes. The three provided sizes of insoles proved to be adequate amounts of alternatives for selecting a suitable insole for each subject. Everybody was able to independently adjust the size of the hip belt and the supporting structure, as it was realized in accordance of the use of a backpack via a belt and a buckle, as well as Velcro fasteners. The weight on the distal body segment was not noticeable and all joints were freely movable without feeling that the usual gait behavior was restrained. The weight of the hip belt was also reported to be appropriate, but might be reduced, due 
to the compressed air tank (highest weight of all system components) that was applied for an autonomous functionality.

The users experienced a simple and intuitive operation of the system, as there is only an on/off switch to start the supporting function. During walking with the exosuit, the dynamic of the assistive force proved to be fast and high enough to lift the foot during swing phase while walking with self-selected, usual gait speed. The insole integrated force sensors detected the gait events in a reliable manner and the control device provided a rapid output to initiate the pneumatic valves without noticeable delay. Hereby, the system also deactivated the actuation appropriately, so that it was possible to freely lower the foot during the initial stance phase (loading response). In summary, the test subjects reported a "natural" feeling while walking with the exosuit.

\section{Discussion}

\subsection{Exosuit Design}

The purpose of the design approach was to develop a soft and lightweight exosuit, which provides a comfortable wear and actuates the ankle joint while walking. The basic design of human-system and hardware interfaces combined with the flow of forces using artificial tendons proved to be suitable to actuate desired joints. The development of the supporting structure is one key achievement of the current exosuit. When intending to apply forces to the body using soft elements, tensile forces are necessary, which need to be counteracted by a non-slip structure. Most exosuits comprise calf wraps $[45,46]$, which have to fit tightly on the entire shank. Considering a prolonged use, the surrounding of a body segment using textiles might lead to increased transpiration followed by friction and skin irritations $[47,48]$. A smaller area of the human-system interface is possible by transmitting the counterforces onto the muscle body of the $\mathrm{m}$. triceps surea. Nevertheless, the interface needs to be large enough to avoid local pressure points. The used webbing with a width of $5 \mathrm{~cm}$ in combination with anti-slip pads maintains an appropriate wearing comfort. Moreover, the slim-line design allows the user to wear the device "invisibly" underneath the clothes, which is an important aspect for acceptance, regarding the usage of an aid in public [49]. Nevertheless, a pneumatic actuation system is not always very applicable for everyday life. It provides major advantages, such as excellent dynamic properties, which are essential for a simultaneous supporting function during gait. To overcome the constraint of being reliant on pressurized air, an electro motor with adequate dynamics could be an opportunity and is planned for further development. To keep the weight of the system as low as possible, a twisted string actuation module was shown to be a reliable and lightweight method for activating distal body segments by translating rotational force into desired tensile force [50].

Although this exosuit was designed with a focus on patients suffering from a dorsiflexion weakness, it could also be used to assist a plantarflexion weakness by adjusting the control algorithm. Moreover, a modification of some parts of the exosuit is conceivable to actuate different joints. For example, to actuate the knee joint, the basic design concept could be used by adjusting the supporting structure to fit at the shank and the thigh. This transfer of the concept to assist different neurological impairments could lead to a system that comprises various modules, which can be combined depending on the desired use.

\subsection{Influence on Gait Kinematics and Muscle Activity}

The purpose of the preliminary evaluation was to identify any restriction in the degrees of freedom for the ankle while walking with the exosuit in comparison to a non-assisted healthy gait. Moreover, a possible reduction in muscle activation by using the exosuit should be analyzed. We hypothesized that there are no restrictions at the ankle kinematics due to the soft structure of the exosuit and the high dynamics of the pneumatic actuation.

The results show that the angle curves of the ankle in all three planes and of the knee and hip joint in the sagittal plane do not differ from a healthy gait when walking with the assistance of the exosuit. The null hypothesis was therefore not rejected, as hypothesized. 
Because of the retention of the analyzed gait kinematics, the exosuit could be suitable for use by patients with dorsiflexion weakness without restraining plantarflexion or introducing negative effects on gait and joint kinetics like passive orthosis does [14]. Hence, the exosuit may be an appropriate solution for gait training with neurological impairment.

For rehabilitation of patients suffering from drop foot, optimized training enhancing the unhealthy gait characteristics including compensation mechanisms such as hip hiking and circumduction is targeted. Since it is a neuromuscular disease, the goal of the corresponding training is to stimulate an afferent input regarding a healthy gait pattern, which in turn could activate efferent motor pathways and thereby retrain the addressed muscles in a positive manner [51-53]. Moreover, activating and using the weakened muscles counteracts muscle atrophy. As it is highly relevant for patients to train and activate dorsiflexion muscles, a reduction in muscle activity while using any assistive device would be counterproductive. The risk of "passive movements" and therefore reduced muscle activity could not be seen while walking with the current exosuit. There was no significant difference in muscle activation of the dorsiflexion muscle tibialis anterior during the walk. Hence, the provided support during the concentric activation phase of $\mathrm{m}$. tibialis anterior seems not to lead to a different motor adaption, which is in accordance with the results of other actively powered ankle devices [54]. The current developed exosuit might have the potential to improve rehabilitation training on the one hand and facilitate walking in the daily routine on the other hand. As a result, an independent and self-reliant gait with a lower risk of a drop foot-related stumbling and falling is aspired to. Therefore, the next step for evaluating the exosuit is to have patients with drop foot test it. Besides the increase in the toe clearance at swing phase, spatiotemporal parameters such as step length and step width, as well as their symmetry between left and right leg, should also be taken into account.

Methodically, SPM1d is preferable for evaluating the effects of assistive devices to one-dimensional biomechanical data such as kinematics, kinetics and EMG. Regarding a holistic approach, SPM allows us to examine the whole time-series data without focusing on special discrete parameters such as maximum or minimum values, which would disregard important information. The used method is able to ensure the current subgoal of the iterative development and was necessary before proceeding to the next step of evaluation. Nevertheless, there are some limitations regarding the current study. The familiarization period of five minutes seems to be too short, due to the noticeable greater variance during the exosuit walking condition. Especially regarding a following analysis of patients, they should have more time to discover the assistive device for achieving a steady state while walking with the system. Furthermore, joints of the contralateral leg should be taken into account when interpreting the effect of an exosuit by doing a gait analysis. This also requires a larger number of subjects. Moreover, the signals of the FSR sensors should be synchronized and recorded with the kinematic measurement, in order to show the signal together with the joint trajectories and to ensure the correct determination of the gait events.

\section{Conclusions}

In conclusion, we presented the design of an innovative exosuit for support of ankle dorsiflexion, which is lightweight and provides good adaptability to varying body dimensions due to soft and flexible materials. A preliminary evaluation examined the biomechanical performance with neurologically intact persons as a first step towards clinical translation. The exosuit provides autonomous support during swing phase, without inhibiting other phases of gait like passive devices mostly do. These insights can be used to guide future studies on patients suffering from dorsiflexion weakness.

Author Contributions: Conceptualization and methodology, T.S. and R.W.; investigation, T.S.; writing - original draft preparation, T.S.; writing — review and editing, R.W. and B.W.; visualization, T.S.; supervision, R.W. and B.W. All authors have read and agreed to the published version of the manuscript. 
Funding: This publication has been funded by the Open-Access-Fund of the Helmut-SchmidtUniversity and by the Federal Ministry for Economic Affairs and Energy (BMWi), grant number 16KN048251.

Institutional Review Board Statement: The study was conducted according to the guidelines of the Declaration of Helsinki and approved by the Ethics Committee of the Department of Psychology of the Helmut-Schmidt University.

Informed Consent Statement: Informed consent was obtained from all subjects involved in the study.

Data Availability Statement: All data are presented in the manuscript.

Conflicts of Interest: The authors declare no conflict of interest.

\section{References}

1. Li, S.; Francisco, G.E.; Zhou, P. Post-stroke Hemiplegic Gait: New Perspective and Insights. Front. Physiol. 2018, 9, 1021. [CrossRef]

2. Chisholm, A.E.; Perry, S.D.; McIlroy, W.E. Correlations between ankle-foot impairments and dropped foot gait deviations among stroke survivors. Clin. Biomech. 2013, 28, 1049-1054. [CrossRef] [PubMed]

3. Błażkiewicz, M.; Wiszomirska, I.; Kaczmarczyk, K.; Brzuszkiewicz-Kuźmicka, G.; Wit, A. Mechanisms of compensation in the gait of patients with drop foot. Clin. Biomech. 2017, 42, 14-19. [CrossRef]

4. Lee, J.H.; An, J.H.; Lee, S.H.; Seo, I.S. Three-dimensional gait analysis of patients with weakness of ankle dorsiflexor as a result of unilateral L5 radiculopathy. J. Back Musculoskelet. Rehabil. 2010, 23, 49-54. [CrossRef] [PubMed]

5. Hussain, S.; Jamwal, P.K.; Ghayesh, M.H. State-of-the-art robotic devices for ankle rehabilitation: Mechanism and control review. Proc. Inst. Mech. Eng. Part H 2017, 231, 1224-1234. [CrossRef]

6. Sarma, J.; Sahai, N.; Bhatia, D. Recent advances on ankle foot orthosis for gait rehabilitation: A review. IJBET 2020, $33,159$. [CrossRef]

7. Pongpipatpaiboon, K.; Mukaino, M.; Matsuda, F.; Ohtsuka, K.; Tanikawa, H.; Yamada, J.; Tsuchiyama, K.; Saitoh, E. The impact of ankle-foot orthoses on toe clearance strategy in hemiparetic gait: A cross-sectional study. J. Neuroeng. Rehabil. 2018, 15, 41. [CrossRef]

8. Cakar, E.; Durmus, O.; Tekin, L.; Dincer, U.; Kiralp, M.Z. The ankle-foot orthosis improves balance and reduces fall risk of chronic spastic hemiparetic patients. Eur. J. Phys. Rehabil. Med. 2010, 46, 363-368.

9. Ramstrand, N.; Ramstrand, S. AAOP State-of-the-Science Evidence Report: The Effect of Ankle-Foot Orthoses on Balance-A Systematic Review. JPO J. Prosthet. Orthot. 2010, 22, P4-P23. [CrossRef]

10. Pourhoseingholi, E.; Saeedi, H. Role of the newly designed Ankle Foot Orthosis on balance related parametersin drop foot post stroke patients. J. Bodyw. Mov. Ther. 2021, 26, 501-504. [CrossRef] [PubMed]

11. Yamamoto, M.; Shimatani, K.; Hasegawa, M.; Kurita, Y. Effect of an ankle-foot orthosis on gait kinematics and kinetics: Case study of post-stroke gait using a musculoskeletal model and an orthosis model. Robomech. J. 2019, 6, 550. [CrossRef]

12. Yeung, L.-F.; Ockenfeld, C.; Pang, M.-K.; Wai, H.-W.; Soo, O.-Y.; Li, S.-W.; Tong, K.-Y. Randomized controlled trial of robot-assisted gait training with dorsiflexion assistance on chronic stroke patients wearing ankle-foot-orthosis. J. Neuroeng. Rehabil. 2018, 15, 51. [CrossRef]

13. Tyson, S.F.; Vail, A.; Thomas, N.; Woodward-Nutt, K.; Plant, S.; Tyrrell, P.J. Bespoke versus off-the-shelf ankle-foot orthosis for people with stroke: Randomized controlled trial. Clin. Rehabil. 2018, 32, 367-376. [CrossRef] [PubMed]

14. Vistamehr, A.; Kautz, S.A.; Neptune, R.R. The influence of solid ankle-foot-orthoses on forward propulsion and dynamic balance in healthy adults during walking. Clin. Biomech. 2014, 29, 583-589. [CrossRef] [PubMed]

15. Bowden, M.G.; Balasubramanian, C.K.; Neptune, R.R.; Kautz, S.A. Anterior-posterior ground reaction forces as a measure of paretic leg contribution in hemiparetic walking. Stroke 2006, 37, 872-876. [CrossRef]

16. Dobkin, B.H.; Plummer-D'Amato, P.; Elashoff, R.; Lee, J. International randomized clinical trial, stroke inpatient rehabilitation with reinforcement of walking speed (SIRROWS), improves outcomes. Neurorehabil. Neural Repair 2010, 24, 235-242. [CrossRef] [PubMed]

17. Schmid, A.; Duncan, P.W.; Studenski, S.; Lai, S.M.; Richards, L.; Perera, S.; Wu, S.S. Improvements in speed-based gait classifications are meaningful. Stroke 2007, 38, 2096-2100. [CrossRef]

18. Altschuck, N.; Bauer, C.; Nehring, I.; Böhm, H.; Jakobeit, M.; Schröder, A.S.; Mall, V.; Jung, N.H. Efficacy of prefabricated carbon-composite ankle foot orthoses for children with unilateral spastic cerebral palsy exhibiting a drop foot pattern. J. Pediatr. Rehabil. Med. 2019, 12, 171-180. [CrossRef]

19. Chen, B.; Zi, B.; Zeng, Y.; Qin, L.; Liao, W.-H. Ankle-foot orthoses for rehabilitation and reducing metabolic cost of walking: Possibilities and challenges. Mechatronics 2018, 53, 241-250. [CrossRef]

20. Kubasad, P.R.; Gawande, V.A.; Todeti, S.R.; Kamat, Y.D.; Vamshi, N. Design and analysis of a passive ankle foot orthosis by using transient structural method. J. Phys. Conf. Ser. 2020, 1706, 12203. [CrossRef]

21. Carolus, A.E.; Becker, M.; Cuny, J.; Smektala, R.; Schmieder, K.; Brenke, C. The Interdisciplinary Management of Foot Drop. Dtsch. Arztebl. Int. 2019, 116, 347-354. [CrossRef] 
22. Huo, W.; Mohammed, S.; Amirat, Y.; Kong, K. Fast Gait Mode Detection and Assistive Torque Control of an Exoskeletal Robotic Orthosis for Walking Assistance. IEEE Trans. Robot. 2018, 34, 1035-1052. [CrossRef]

23. Kirtas, O.; Savas, Y.; Bayraker, M.; Baskaya, F.; Basturk, H.; Samur, E. Design, implementation, and evaluation of a backstepping control algorithm for an active ankle-foot orthosis. Control Eng. Pract. 2021, 106, 104667. [CrossRef]

24. Hamedi, M.; Salimi, P.; Aliabadi, A.; Vismeh, M. Toward intelligent ankle foot orthosis for foot-drop, a review of technologies and possibilities. In Proceedings of the 2nd International Conference on Biomedical Engineering (ICoBE), Penang, Malaysia, 30-31 March 2015; IEEE: Piscataway, NJ, USA, 2015; pp. 1-6, ISBN 978-1-4799-1749-5.

25. Yeung, L.-F.; Ockenfeld, C.; Pang, M.-K.; Wai, H.-W.; Soo, O.-Y.; Li, S.-W.; Tong, K.-Y. Design of an exoskeleton ankle robot for robot-assisted gait training of stroke patients. IEEE Int. Conf. Rehabil. Robot. 2017, 2017, 211-215. [CrossRef] [PubMed]

26. Browning, R.C.; Modica, J.R.; Kram, R.; Goswami, A. The effects of adding mass to the legs on the energetics and biomechanics of walking. Med. Sci. Sports Exerc. 2007, 39, 515-525. [CrossRef] [PubMed]

27. Abe, I.; Ishiya, K.; Oshimoto, T.; Kikuchi, T.; Tanida, S.; Yasuda, T. Ankle-foot orthosis using elastomer-embedded flexible joint. Annu. Int. Conf. IEEE Eng. Med. Biol. Soc. 2017, 2017, 4499-4502. [CrossRef]

28. Awad, L.N.; Kudzia, P.; Revi, D.A.; Ellis, T.D.; Walsh, C.J. Walking faster and farther with a soft robotic exosuit: Implications for post-stroke gait assistance and rehabilitation. IEEE Open J. Eng. Med. Biol. 2020, 1, 108-115. [CrossRef]

29. Ding, Y.; Galiana, I.; Asbeck, A.T.; de Rossi, S.M.M.; Bae, J.; Santos, T.R.T.; de Araujo, V.L.; Lee, S.; Holt, K.G.; Walsh, C. Biomechanical and Physiological Evaluation of Multi-Joint Assistance with Soft Exosuits. IEEE Trans. Neural Syst. Rehabil. Eng. 2017, 25, 119-130. [CrossRef]

30. Tucker, M.R.; Olivier, J.; Pagel, A.; Bleuler, H.; Bouri, M.; Lambercy, O.; Millán, J.D.R.; Riener, R.; Vallery, H.; Gassert, R. Control strategies for active lower extremity prosthetics and orthotics: A review. J. Neuroeng. Rehabil. 2015, 12, 1. [CrossRef]

31. Yuan, K.; Wang, Q.; Wang, L. Fuzzy-Logic-Based Terrain Identification with Multisensor Fusion for Transtibial Amputees. IEEE ASME Trans. Mechatron. 2015, 20, 618-630. [CrossRef]

32. Gmerek, A.; Meskin, N.; Tehrani, E.S.; Kearney, R. Design of a hydraulic ankle-foot orthosis. In Proceedings of the 6th IEEE RAS/EMBS International Conference, Singapore, 26-29 June 2016; pp. 1041-1048.

33. Arnez-Paniagua, V.; Rifai, H.; Mohammed, S.; Amirat, Y. Adaptive Control of an Actuated Ankle Foot Orthosis for Foot-Drop Correction. IFAC PapersOnLine 2017, 50, 1384-1389. [CrossRef]

34. Gmerek, A.; Davoodi, M.; Meskin, N.; Tehrani, E.S.; Kearney, R.E. A new ankle foot orthosis: Modeling and control. In Proceedings of the 4th International Conference on Control, Decision and Information Technologies (CoDIT), Barcelona, Spain, 5-7 April 2017; pp. 1066-1071.

35. Dzahir, M.; Yamamoto, S. Recent Trends in Lower-Limb Robotic Rehabilitation Orthosis: Control Scheme and Strategy for Pneumatic Muscle Actuated Gait Trainers. Robotics 2014, 3, 120-148. [CrossRef]

36. Lairamore, C.; Garrison, M.K.; Bandy, W.; Zabel, R. Comparison of tibialis anterior muscle electromyography, ankle angle, and velocity when individuals post stroke walk with different orthoses. Prosthet. Orthot. Int. 2011, 35, 402-410. [CrossRef] [PubMed]

37. Kubasad, P.R.; Todeti, S.R.; Kamat, Y.D. A Review on Designs of Various Ankle Foot Orthosis (AFO) Used to Treat Drop Foot Disease. In Mechanism and Machine Science; Sen, D., Mohan, S., Ananthasuresh, G.K., Eds.; Springer: Singapore, 2021 ; pp. 789-807. ISBN 978-981-15-4476-7.

38. Hollman, J.H.; McDade, E.M.; Petersen, R.C. Normative spatiotemporal gait parameters in older adults. Gait Posture 2011, 34, 111-118. [CrossRef] [PubMed]

39. Quinlivan, B.; Asbeck, A.; Wagner, D.; Ranzani, T.; Russo, S.; Walsh, C. Force Transfer Characterization of a Soft Exosuit for Gait Assistance. In International Design Engineering Technical Conferences and Computers and Information in Engineering; The American Society of Mechanical Engineers: New York, NY, USA, 2016; ISBN 978-0-7918-5712-0.

40. Ledoux, W.R. The Biomechanics of Aging and Diabetic Plantar Soft Tissue. In Mechanical Properties of Aging Soft Tissues; Derby, B., Akhtar, R., Eds.; Springer International Publishing: Cham, Switzerland, 2015; pp. 187-206. ISBN 978-3-319-03969-5.

41. Seel, T.; Raisch, J.; Schauer, T. IMU-based joint angle measurement for gait analysis. Sensors 2014, 14, 6891-6909. [CrossRef] [PubMed]

42. Al-Amri, M.; Nicholas, K.; Button, K.; Sparkes, V.; Sheeran, L.; Davies, J.L. Inertial Measurement Units for Clinical Movement Analysis: Reliability and Concurrent Validity. Sensors 2018, 18, 719. [CrossRef]

43. Sorbie, G.G.; Williams, M.J.; Boyle, D.W.; Gray, A.; Brouner, J.; Gibson, N.; Baker, J.S.; Easton, C.; Ugbolue, U.C. Intra-session and Inter-day Reliability of the Myon 320 Electromyography System During Sub-maximal Contractions. Front. Physiol. 2018, 9, 309. [CrossRef] [PubMed]

44. Pataky, T.C. One-dimensional statistical parametric mapping in Python. Comput. Methods Biomech. Biomed. Eng. 2012, 15, $295-301$. [CrossRef]

45. Malcolm, P.; Lee, S.; Crea, S.; Siviy, C.; Saucedo, F.; Galiana, I.; Panizzolo, F.A.; Holt, K.G.; Walsh, C.J. Varying negative work assistance at the ankle with a soft exosuit during loaded walking. J. Neuroeng. Rehabil. 2017, 14, 62. [CrossRef]

46. Bae, J.; Siviy, C.; Rouleau, M.; Menard, N.; Odonnell, K.; Geliana, I.; Athanassiu, M.; Ryan, D.; Bibeau, C.; Sloot, L.; et al. A Lightweight and Efficient Portable Soft Exosuit for Paretic Ankle Assistance in Walking After Stroke. In Proceedings of the 2018 IEEE International Conference on Robotics and Automation (ICRA), Brisbane, Australia, 21-25 May 2018; Lynch, K., Ed.; IEEE: Piscataway, NJ, USA, 2018; pp. 2820-2827, ISBN 978-1-5386-3081-5. 
47. Wollina, U. Mechanical skin irritations due to textiles. In Handbook of Medical Textiles; Bartels, V.T., Ed.; Woodhead Pub: Oxford, UK, 2011; pp. 248e-268e. ISBN 978-1-84569-691-7.

48. Quintero-Quiroz, C.; Pérez, V.Z. Materials for lower limb prosthetic and orthotic interfaces and sockets: Evolution and associated skin problems. Rev. Fac. Med. 2019, 67, 117-125. [CrossRef]

49. Shore, L.; Power, V.; De Eyto, A.; O'Sullivan, L. Technology Acceptance and User-Centred Design of Assistive Exoskeletons for Older Adults: A Commentary. Robotics 2018, 7, 3. [CrossRef]

50. Palli, G.; Natale, C.; May, C.; Melchiorri, C.; Wurtz, T. Modeling and Control of the Twisted String Actuation System. IEEE ASME Trans. Mechatron. 2013, 18, 664-673. [CrossRef]

51. Willerslev-Olsen, M.; Petersen, T.H.; Farmer, S.F.; Nielsen, J.B. Gait training facilitates central drive to ankle dorsiflexors in children with cerebral palsy. Brain 2015, 138, 589-603. [CrossRef] [PubMed]

52. Li, S. Spasticity, Motor Recovery, and Neural Plasticity after Stroke. Front. Neurol. 2017, 8, 120. [CrossRef]

53. Dietz, V. Locomotor activity in spinal man: Significance of afferent input from joint and load receptors. Brain 2002, 125, 2626-2634. [CrossRef]

54. Kao, P.-C.; Ferris, D.P. Motor adaptation during dorsiflexion-assisted walking with a powered orthosis. Gait Posture 2009, 29, 230-236. [CrossRef] 